\title{
MEASURABLE OR CONDENSING MULTIVALUED MAPPINGS AND RANDOM FIXED POINT THEOREMS
}

\author{
By SHIGERU ITOH
}

\section{Introduction}

Various results on random fixed point theorems were given by many authors (cf. Bharucha-Reid [1, 2], Itoh [7, 8], Engl $[3,4]$ and their references). In [8] almost all known fixed point theorems (e.g. for nonexpansive or condensing mappings) were extended to random cases (except for contraction mappings that is due to Špaček [16] and Hanš [5]) on general measurable spaces. Similar results were obtained by Bharucha-Reid [2] and Engl [3, 4] on measure spaces.

For multivalued mappings, a random fixed point theorem for contraction mappings was proved in [7]. Then in [8], theorems for multivalued condensing or nonexpansive mappings on measurable spaces were treated, where in the former case lower semicontinuity as well as upper semicontinuity are assumed. On measure spaces, Engl $[3,4]$ gave a theorem which makes possible to derive random fixed point theorems from fixed point theorems for multivalued continuous (in Hausdorff metric) mappings. Moreover, he obtained a complete result of Bohnenblust and Karlin type for upper semicontinuous compact multivalued mappings.

Other results on random equations were treated by Kannan and Salehi [11] and Itoh $[9,10]$.

In this paper, by adopting the method of Engl $[3,4]$ we prove random fixed point theorems for upper semicontinuous condensing multivalued mappings. In sections 1 and 2, some results on upper semicontinuity and measurability of multivalued mappings are presented. Then in section 3 random fixed point theorems are given.

\section{Upper Semicontinuous Multivalued Mappings}

Let $X$ be a metric space. For any $B \subset X$ and $p>0$, let $\operatorname{cl}(B)$ be the closure of $B$ and $B_{p}=\{x \in X: d(x, B)<p\}$, where $d(x, B)=\inf \{d(x, y): y \in B\}$. Let $2^{X}$ be the family of all subsets of $X, C D(X)$ all nonempty closed subsets, and $K(X)$ all nonempty compact subsets of $X$ respectively. If $X$ is a subset of a Banach

Received May 4, 1978 
space, denote by $\operatorname{clco}(X)$ the closed convex hull of $X$ and by $C K(X)$ the family of all nonempty compact convex subsets of $X$. Let $Y$ be another metric space. A mapping $F: X \rightarrow C D(Y)$ is said to be upper semucontinuous (u.s.c.) if for any closed subset $C$ of $Y, F^{-1}(C)=\{x \in X: F(x) \cap C \neq \emptyset\}$ is a closed subset of $X$. It is obvious that $F$ is u. s. c. if and only if given $x \in X$, for each open subset $V$ of $Y$ with $V \supset F(x)$, there exists a neighborhood $U$ of $x$ such that $F(y) \subset V$ whenever $y \in U$.

LEMMA 1.1. Let $X$ be a separable metric space with $\left\{x_{k}\right\}$ a countable dense subset of $X$ and $Y$ be a Banach space. Let $F: X \rightarrow C K(Y)$ be an u.s.c. mapping, then the mapping $G: X \rightarrow 2^{Y}$ defined by

$$
G(x)=\bigcap_{n=1}^{\infty} \operatorname{clco}\left(\cup\left\{F\left(x_{k}\right): d\left(x_{k}, x\right)<1 / n\right\}\right) \quad(x \in X)
$$

satısfies the conditions:

(i) For any $x \in X, F(x) \supset G(x) \neq \emptyset$.

(ii) $G$ is u.s.c.

Proof. For each $n$, define $G_{n}: X \rightarrow C D(Y)$ by

then

$$
G_{n}(x)=\operatorname{clco}\left(\cup\left\{F\left(x_{k}\right): d\left(x_{k}, x\right)<1 / n\right\}\right) \quad(x \in X),
$$

$$
G(x)=\bigcap_{n=1}^{\infty} G_{n}(x) .
$$

We first show that $G(x)$ is nonempty for every $x \in X$. For any $n$, take $k_{n}$ such that $d\left(x_{k_{n}}, x\right)<1 / n$. Then

$$
G_{n}(x) \supset \operatorname{clco}\left(\bigcup_{\imath \geqq n} F\left(x_{k_{i}}\right)\right) .
$$

Since $\left\{x_{k_{n}}\right\}_{n=1}^{\infty} \cup\{x\}$ is compact and $F$ is u. s. c., $\bigcup_{n=1}^{\infty} F\left(x_{k_{n}}\right) \cup F(x)$ is compact, hence $\operatorname{clco}\left(\bigcup_{\imath \geqq n} F\left(x_{k_{i}}\right)\right)$ is compact. Thus

$$
G(x)=\bigcap_{n=1}^{\infty} G_{n}(x) \supset \bigcap_{n=1}^{\infty} \operatorname{clco}\left(\bigcup_{\imath \geqq n} F\left(x_{k_{i}}\right)\right) \neq \emptyset .
$$

The relation $F(x) \supset G(x)$ is an easy consequence of $F$ being u. s. c. Indeed, for any $p>0$, take $n$ for sufficiently large, then $d\left(x_{k}, x\right)<1 / n$ implies $F\left(x_{k}\right) \subset(F(x))_{p}$. Since $(F(x))_{p}$ is convex, $G(x) \subset G_{n}(x) \subset \operatorname{cl}\left((F(x))_{p}\right)$, which yields $G(x) \subset F(x)$, Now we prove that $G$ is u.s.c. Let $C$ be any closed subset of $Y$ and $\left\{z_{i}\right\}$ be a sequence of $G^{-1}(C)$ converging to some $z \in X$. For each $n$, choose $z_{2}$ such that $d\left(z_{\imath}, z\right)<1 / 2 n$. If $d\left(x_{k}, z_{\imath}\right)<1 / 2 n$, then $d\left(x_{k}, z\right)<1 / n$, hence $G_{2 n}\left(z_{\imath}\right) \subset G_{n}(z)$ and $G_{n}(z) \cap C \supset G_{2 n}\left(z_{\imath}\right) \cap C \neq \emptyset$. Since $F$ is u.s. c., there exists $j_{n}>n$ for which $d\left(x_{k}, z\right)$ $<1 / \jmath_{n}$ implies $F\left(x_{k}\right) \subset(F(z))_{1 / n}$. Thus $\emptyset \neq G_{\jmath_{n}}(z) \cap C \subset \operatorname{cl}\left((F(z))_{1 / n}\right)$. There exists $y_{n} \in G_{\jmath_{n}}(z) \cap C$ such that $d\left(y_{n}, F(z)\right) \leqq 1 / n$. Since $F(z)$ is compact, some subsequence $\left\{y_{m}\right\}$ of $\left\{y_{n}\right\}$ converges to an element $y$ of $C$. If $j_{m}>n$, then $y_{m} \in$ 
$G_{\jmath_{m}}(z) \cap C \subset G_{n}(z) \cap C$. It follows that $y \in G_{n}(z) \cap C$ for all $n$. This implies

$$
y \in \bigcap_{n=1}^{\infty} G_{n}(z) \cap C=G(z) \cap C,
$$

and $z \in G^{-1}(C)$. Hence $G^{-1}(C)$ is closed and $G$ is u. s.c.

Remark 1.2. Almost the same proof as above also establishes the following: Let $X$ be a separable metric space with $\left\{x_{k}\right\}$ a countable dense subset of $X, Y$ be a metric space, and $F: X \rightarrow K(Y)$ be u.s.c. Then the mapping $G: X \rightarrow 2^{Y}$ by

$$
G(x)=\bigcap_{n=1}^{\infty} \operatorname{cl}\left(\cup\left\{F\left(x_{k}\right): d\left(x_{k}, x\right)<1 / n\right\}\right) \quad(x \in X)
$$

has the properties:

(i) For any $x \in X, F(x) \supset G(x) \neq \emptyset$.

(ii) $G$ is upper semicontinuous.

\section{§2. Measurable Multivalued Mappings}

In the sequel, let $(T, \mathcal{A})$ be a measurable space. A mapping $F: T \rightarrow 2^{X}$ is said to be (A)-measurable if for each closed subset $C$ of $X, F^{-1}(C)=\{t \in T: F(t)$ $\cap C \neq \emptyset\} \in \mathcal{A} . \quad F$ is said to be (A-)weakly measurable if for each open subset $B$ of $X, F^{-1}(B) \in \mathcal{A}$. It is obvious that if $F$ is measurable, then $F$ is weakly measurable. If $F(t) \in K(X)$ for all $t \in T$, then the converse is valid by Himmelberg [6, Theorem 3.1]. See also Wagner [17]. Denote by $\mathscr{B}$ the Borel field of $X$ and by $\mathcal{A} \times \mathscr{B}$ the product $\sigma$-algebra of $\mathcal{A}$ and $\mathscr{B}$ on $T \times X$.

Proposition 2.1. Let $X$ be a separable metric space with $\left\{x_{k}\right\}$ a countable dense subset of $X$ and $Y$ be a separable Banach space. Let $F: T \times X \rightarrow C K(Y)$ be a mapping having the properties:

(a) For each $t \in T, F(t, \cdot)$ is u.s.c.

(b) For each $x \in X, F(\cdot, x)$ is weakly measurable.

Then the mapping $G: T \times X \rightarrow 2^{Y}$ defined by

$$
G(t, x)=\bigcap_{n=1}^{\infty} \operatorname{clco}\left(\cup\left\{F\left(t, x_{k}\right): d\left(x_{k}, x\right)<1 / n\right\}\right)
$$

$(t \in T, x \in X)$ satısfies the following conditions:

(i) For each $t \in T$ and $x \in X, F(t, x) \supset G(t, x) \neq \emptyset$.

(ii) For each $t \in T, G(t, \cdot)$ is u.s.c.

(iii) $G$ is $\mathcal{A} \times \mathscr{B}$-measurable.

Proof. (i) and (ii) is clear from Lemma 1.1.

(iii) For each $n$, define $H_{n}: T \times X \rightarrow 2^{Y}$ by $H_{n}(t, x)=\cup\left\{F\left(t, x_{k}\right): d\left(x_{k}, x\right)<1 / n\right\}$ $(t \in T, x \in X)$, then $H_{n}$ is $\mathcal{A} \times \mathscr{B}$-weakly measurable. Indeed, for any open subset $B$ of $Y$, 


$$
\begin{aligned}
H_{n}{ }^{-1}(B) & =\left\{(t, x) \in T \times X: H_{n}(t, x) \cap B \neq \emptyset\right\} \\
& =\bigcup_{k=1}^{\infty}\left\{t \in T: F\left(t, x_{k}\right) \cap B \neq \emptyset\right\} \times\left\{x \in X: d\left(x, x_{k}\right)<1 / n\right\} \in \mathcal{A} \times \mathscr{B} .
\end{aligned}
$$

Then the mapping $G_{n}: T \times X \rightarrow C D(Y)$ defined by $G_{n}(t, x)=\operatorname{clco}\left(H_{n}(t, x)\right)$ is $\mathcal{A} \times \mathscr{B}$ weakly measurable by Himmelberg [6, Theorem 9.1]. If we show that

$$
G^{-1}(C)=\bigcap_{n=1}^{\infty} G_{n}^{-1}\left(C_{1 / n}\right)
$$

for every closed subset $C$ of $Y$, then we can conclude that $G$ is $\mathcal{A} \times \mathscr{B}$-measurable. It is obvious that

$$
G^{-1}(C) \subset \bigcap_{n=1}^{\infty} G_{n}^{-1}\left(C_{1 / n}\right)
$$

Conversely, if

$$
(t, x) \in \bigcap_{n=1}^{\infty} G_{n}{ }^{-1}\left(C_{1 / n}\right),
$$

then $G_{n}(t, x) \cap C_{1 / n} \neq \emptyset$ for all $n$. Since $F(t, \cdot)$ is u.s.c., by the same way as in the proof of Lemma 1.1 we have

$$
\emptyset \neq \bigcap_{n=1}^{\infty} G_{n}(t, x) \cap \operatorname{cl}\left(C_{1 / n}\right)=G(t, x) \cap C .
$$

Hence

$$
\bigcap_{n=1}^{\infty} G_{n}^{-1}\left(C_{1 / n}\right) \subset G^{-1}(C)
$$

Remark 2.2. Let $F$ be as in Proposition 2.1, then $F$ itself is not necessarily $\mathcal{A} \times \mathscr{B}$-measurable (cf. Engl $[3,4]$ ).

Remark 2.3. By a slight modification of the above proof we can prove the following: Let $X$ be a separable metric space with $\left\{x_{k}\right\}$ a countable dense subset of $X$ and $Y$ be a metric space. Let $F: T \times X \rightarrow K(Y)$ be a mapping with the properties:

(a) For each $t \in T, F(t, \cdot)$ is u. s.c.

(b) For each $x \in X, F(\cdot, x)$ is weakly measurable. Define $G: T \times X \rightarrow K(Y)$ by

$$
G(t, x)=\bigcap_{n=1}^{\infty} \operatorname{cl}\left(\cup\left\{F\left(t, x_{k}\right): d\left(x_{k}, x\right)<1 / n\right\}\right)
$$

$(t \in T, x \in X)$, then $G$ fulfills the conditions :

(i) For each $t \in T, x \in X, F(t, x) \supset G(t, x) \neq \emptyset$.

(ii) For each $t \in T, G(t, \cdot)$ is u.s.c.

(iii) $G$ is $A \times \mathscr{B}$-measurable.

If $X$ is also complete, the proof of the following lemma is essentially contained in [7, Proposition 4]. If $X$ is a separable Banach space, the same is 
obtained in Engl [4] by a different method.

LEMMA 2.4. Let $X$ be a separable metruc space, $F: T \rightarrow C D(X)$ be a weakly measurable mapping, and $u: T \rightarrow X$ be a measurable mapping. Then $d(u(\cdot), F(\cdot))$ is a measurable function on $T$.

Proof. Define $f: T \times X \rightarrow R$ (the real numbers) by $f(t, x)=d(x, F(t))(t \in T$, $x \in X)$, then $f$ is measurable in $t$ by Himmelberg [6, Theorem 3.3] and continuous in $x$. Hence the function $f(\cdot, u(\cdot))=d(u(\cdot), F(\cdot))$ on $T$ is measurable (cf. Himmelberg [6, Theorem 6.5]).

\section{$\S 3$. Random Fixed Point Theorems}

Let $\Sigma$ and $\Sigma^{*}$ be the respective sets of infinite and finite sequences of positive integers. For $\sigma \in \Sigma$, denote $\left(\sigma_{1}, \cdots, \sigma_{n}\right)$ by $\sigma \mid n$ and let $A: \Sigma^{*} \rightarrow \mathcal{A}$. Then

$$
\bigcup_{\sigma \in \Sigma} \bigcap_{n=1}^{\infty} A_{\sigma i n}
$$

is said to be obtained from $\mathcal{A}$ by the Souslin operation. $\mathcal{A}$ is called a Souslin family if every set obtained from $\mathcal{A}$ in this way is also in $\mathcal{A}$. If there exists a complete $\sigma$-finite measure on $(T, \mathcal{A}$ ), then $\mathcal{A}$ is a Souslin family (cf. Wagner $[17$, p. 864$]$ and the references cited there).

For any bounded subset $B$ of $X$, let $\gamma(B)=\inf \{c>0: B$ can be covered by a finite number of subsets of diameters less than or equal to $c\}$. A mapping $F: X \rightarrow C D(X)$ is said to be condensing if for any bounded subset $B$ of $X$ with $\gamma(B)>0, \gamma(F(B))<\gamma(B)$, where $F(B)=\cup\{F(x): x \in B\}$.

Now we prove the following random fixed point theorem by using the results in section 2 .

THEOREM 3.1. Let $\mathcal{A}$ be a Souslin family and $X$ be a nonempty closed convex subset of a separable Banach space $Y$. Let $F: T \times X \rightarrow C K(Y)$ be a mapping satisfying the conditrons.

(i) For any $t \in T, F(t, X)$ is bounded and $F(t, b d X) \subset X$, where $b d X$ is the boundary of $X$.

(ii) For any $t \in T, F(t, \cdot)$ is u.s.c. and condensing.

(iii) For any $x \in X, F(\cdot, x)$ is weakly measurable.

Then there exists a measurable mapping $u: T \rightarrow X$ such that $u(t) \in F(t, u(t))$ for all $t \in T$.

Proof. Choose countable dense elements $\left\{x_{k}\right\}$ of $X$ and define $G: T \times X \rightarrow$ $C K(Y)$ as in Proposition 2.1, then $G$ is $\mathcal{A} \times \mathscr{B}$-measurable. The mapping $v: T \times X \rightarrow X$ by $v(t, x)=x(t \in T, x \in X)$ is $\mathcal{A} \times \mathscr{B}$-measurable. By Lemma 1.3, $f(t, x)=d(v(t, x), G(t, x))(t \in T, x \in X)$ is a $(\mathcal{A} \times \mathscr{B}$-)measurable function on $T \times X$. Define $H: T \rightarrow 2^{X}$ by $H(t)=\{x \in X: x \in G(t, x)\} \quad(t \in T)$, then for any $t \in T, H(t)$ is 
nonempty and compact by Petryshyn and Fitzpatrick [14] and the method of the proof of Smart [15, Theorem 9.2.4]. Moreover we have

$$
\begin{aligned}
\operatorname{Gr} H & =\{(t, x) \in T \times X: x \in H(t)\} \\
& =\{(t, x) \in T \times X: f(t, x)=0\} \\
& \in \mathcal{A} \times \mathscr{B} .
\end{aligned}
$$

By Leese [12] (cf. Wagner [17, Theorem 4.2]) there exists a measurable mapping $u: T \rightarrow X$ such that for each $t \in T, u(t) \in H(t)$, hence $u(t) \in G(t, u(t)) \subset F(t, u(t))$.

COROllary 3.2. Let $(T, \mathcal{A}, m)$ be a (complete) $\sigma$-fintte measure space and $Y$, $X$, and $F: T \times X \rightarrow C K(Y)$ be as in Theorem 3.1. Then there exists a measurable mapping $u: T \rightarrow X$ such that $u(t) \in F(t, u(t))$ for $m$-a.e. (all) $t \in T$.

Proof. If $m$ is complete, then $\mathcal{A}$ is a Souslin family and the conclusion follows from Theorem 3.1.

If $m$ is not complete, the usual method of considering the completion of $(T, \mathcal{A}, m)$ easily yields the conclusion. We include the proof for completeness. Let $\left(T, \mathcal{A}^{*}, m^{*}\right)$ be the completion of $(T, \mathcal{A}, m)$. Then by Theorem 3.1 there exists a $\mathcal{A}^{*}$-measurable mapping $v: T \rightarrow X$ for which $v(t) \in F(t, v(t))$ for all $t \in T$. Since $X$ is separable, we may take a countable open base $\left\{B_{n}\right\}$ of $X$. For each $n, v^{-1}\left(B_{n}\right)=A_{n} \cup N_{n}$, where $A_{n} \in \mathcal{A}$ and $N_{n}$ is contained in some $D_{n} \in \mathcal{A}$ with $m\left(D_{n}\right)=0$. Then

$$
D=\bigcup_{n=1}^{\infty} D_{n} \in \mathcal{A}
$$

and $m(D)=0$. Let $u: T \rightarrow X$ be a mapping defined by

$$
u(t)=\left\{\begin{array}{l}
v(t) \text { if } t \in T-D, \text { or } \\
y \text { if } t \in D,
\end{array}\right.
$$

where $y$ is any fixed element of $X$. It is easy to observe that $u$ is $\mathcal{A}$-measurable and $u(t) \in F(t, u(t))$ for every $t \in T-D$.

Remark 3.3. We can also state and prove similar results as above in the case that the domain of $F(t, \cdot)$ is dependent on $t \in T$ as in Engl $[3,4]$. The proofs are almost the same as those given above. We omit the details.

Acknowledgement

The author is grateful to Professors H. Umegaki and W. Takahashi for their valuable advice in preparing this paper. 


\section{REFERENCES}

[1] Bharucha-Reid, A.T., Random integral equations. Academic Press, New York and London (1972).

[2] Bharucha-Reid, A.T., Fixed point theorems in probabilistic analysis. Bull. Amer. Math. Soc. 82 (1976), 641-657.

[3] ENGL, H., Random fixed point theorems. Proceedings on a Symposium on Nonlinear Equations in Abstract Spaces (V. Lakshmikantham, Ed.), Academic Press, (to appear).

[4] ENGL, H., Fixed point theorems for random operators on stochastic domains. Dissertation, Johannes Kepler Unıversität Linz, Austrı (1977).

[5] HANš, O., Reduzierende zufällige Transformatıonen. Czechoslovak Math. J. 7 (1957), 154-158.

[6] Himmelberg, C. J., Measurable relations. Fund. Math. 87 (1975), 53-72.

[7] Ітон, S., A random fixed point theorem for a multivalued contraction mapping. Pacific J. Math. 68 (1977), 85-90.

[8] Iтон, S., Random fixed point theorems with an application to random differential equations in Banach spaces. J. Math. Anal. Appl. (to appear).

[9] Iтон, S., Nonlinear random equations with monotone operators in Banach spaces. Res. Rep. Inf. Sci. No. A-45 (1977).

[10] IтоH, S., Random differential equations associated with accretive operators. Res. Rep. Inf. Sci. No. A-46 (1977).

[11] Kannan, R. and H. Salehi, Random nonlinear equations and monotonic nonlinearities. J. Math. Anal. Appl. 57 (1977), 234-256.

[12] Leese, S. J., Multifunctions of Souslin type. Bull. Austral. Math. Soc. 11 (1974), 395-411.

[13] LeEse, S. J., Multifunctions of Souslin type: Corrigendum. Bull. Austral. Math. Soc. 13 (1975), 159-160.

[14] Petryshyn, W. V. and P. M. Fitzpatrick, Fixed-point theorems for multivalued noncompact inward maps. J. Math. Anal. Appl. 46 (1974), 756-767.

[15] Smart, D. R., Fixed point theorems. Cambridge University Press, London (1974).

[16] ŠpAčEK, A., Zufällige Gleichungen. Czechoslovak Math. J. 7 (1957), 154-158.

[17] WAgner, D.H., Survey of measurable selection theorems. SIAM J. Control Optimization 15 (1977), 859-903.

Department of Information Sciences, Tokyo Institute of Technology. 